Chronic Obstructive Pulmonary Diseases:

Journal of the COPD Foundation

\title{
Journal Club \\ Journal Club-Electronic Cigarettes and Vaping as a Harm Reduction Alternative: Really?
}

\author{
Ron Balkissoon, MD, MSc, DIH, FRCPC ${ }^{1}$
}

\begin{abstract}
Abbreviations: electronic cigarettes, e-cigarettes; chronic obstructive pulmonary disease, COPD; European Respiratory Society, ERS; Food and Drug Administration, FDA

Citation: Balkissoon R. Journal club-electronic cigarettes and vaping as a harm reduction alternative: really? Chronic Obstr Pulm Dis. 2019;6(3):281-291. doi: https://doi.org/10.15326/jcopdf.6.3.2019.0143
\end{abstract}

\section{Denver, Colorado}

\section{Address correspondence to:}

Ron Balkissoon, MD, MSc, DIH, FRCPC

balkissoonr@njhealth.org

\section{Keywords}

chronic obstructive pulmonary disease; COPD; vaping; electronic cigarettes; e-cigarettes; nicotine addiction; JUULing

\section{Introduction}

In the last few months I have seen 2 patients who presented with significant respiratory symptoms, (cough, shortness of breath and chest pain) that they temporally associated with use of electronic cigarettes (e-cigarettes). They were relatively new to e-cigarette use: one was a former cigarette smoker and the other was a never smoker but had a history of asthma. The severity of their symptoms had abated but they still had residual symptoms and were worried about the long-term consequences. When I went to review the literature regarding the latest data on the long-term health risks of using e-cigarettes, I noted an explosion of information being published in just the past few years. It is clear there is a great deal of controversy and concern about the introduction of these products into the marketplace without sufficient information about potential health risks nor adequate regulation over their indications, contents, and the technology used to generate their aerosol. Currently there are regular advertisements for e-cigarettes promoting them as viable and safe alternatives to cigarette smoking. One of the key initial promotions for these devices was as a smoking cessation tool despite an absence of any large double-blinded, randomized placebocontrolled trials to prove any superior efficacy to conventional smoking cessation strategies (such as nicotine replacement, bupropion hydrochloride, or the nicotine receptor partial agonists such as varenicline and counseling). More recently however, since their entry into the marketplace in the United States in 2006, manufacturers, many users, and some tobacco control experts, advocate e-cigarettes as a viable longterm harm reduction alternative to cigarette smoking without any long-term safety data. E-cigarettes have become a multi-billion-dollar industry (U.S. \$ 11.3 billion in 2018) and projections suggest that their sales will surpass combustible cigarettes by $2023 .^{1}$ There are few regulations concerning access to these devices and there is high utilization by teenagers and reports of use by middle school children. ${ }^{2}$ The question has been raised of whether these companies are specifically targeting this population with flavors such as "bubble gum." There is also data to suggest that rather than reducing the risk of teenagers smoking cigarettes, use of e-cigarettes may act as a gateway to young people transitioning to smoking tobacco cigarettes. Recent studies show that adolescents and young adult e-cigarette users are at a 3 times greater risk of starting cigarette smoking compared to those who had never used e-cigarettes. ${ }^{3-8}$ (See abstracts below).

The essential design of e-cigarettes includes an atomizer that uses an electrical current, generated by a battery, to heat a metal coil which aerosolizes the e-liquid conducted from a reservoir to the coil via a wick, typically made of cotton or silica. The user 
presses a button on the device, inhales and a plume of droplets carry the aerosol to the oropharynx and respiratory tree. E-cigarettes have evolved from cigarette look-alikes to new industrial-looking devices that afford greater control with regard to the delivery, content and nature of aerosolization as well as heat of the e-liquid. The typical constituents in most e-liquids are vegetable glycerin, propylene glycol, nicotine, water, alcohol, flavoring agents and a variety of other substances. Some e-liquids actually do not have nicotine in them but still contain various flavoring agents. The assumption has been that the elimination of tobacco tar, smoke particulate and various chemical additives and their combustible by-products renders the e- cigarettes to be a safer alternative. While this may very well be true, it is important to appreciate that the various components of the e-cigarette are not benign and can potentially pose significant health risks and there is no regulation over the content and access to these devices.

Recent studies have outlined potential toxic effects from nicotine (see below) including increased airway hyper-reactivity, inflammation, reduction of host defenses and possible cardiovascular effects. ${ }^{9-14}$ While nicotine is not considered a carcinogen, there are studies to suggest it may potentiate cancer. ${ }^{15,16}$ There have also been recent studies suggesting that chronic obstructive pulmonary disease (COPD) patients may be more susceptible to potential harmful effects of e-cigarettes. ${ }^{17,18}$ There are reports of fatalities in children as young as 5 in which the e-liquids were ingested. ${ }^{19}$ The nicotine content of the e-liquid cartridges continues to be increased and there is 1 brand that offers a single e-liquid cartridge that contains the equivalent nicotine $(47 \mathrm{mg})$ of 20 cigarettes (1 pack). ${ }^{20}$ Nicotine is highly addictive and with the increased stimulation of the central nervous system there is evidence not only that use of these e-cigarettes leads to some never cigarette smokers transitioning to use of tobacco cigarettes (so called "gateway") 3,21,22 but also to the use of illicit drugs such as cannabinoids. ${ }^{14,23}$ Furthermore, studies of quality control have shown that there are inconsistencies between actual nicotine concentrations and those indicated on labeling. $1,12,20,24-28$

There are reported to be over 8000 flavoring agents now used in e-cigarettes. Aromatic aldehyde compounds such as cinnamon flavor, (cinnamon aldehyde), almond flavor, (benzaldehyde), and vanilla flavors (vanilla and ethyl vanilla), as well as formaldehyde, have potential toxic effects when inhaled. $^{2,20,28-30}$ There are reports that some of these flavoring agents have already been associated with bronchiolitis, alveolitis and acute lung injury. 1,31-33 Diacetyl flavoring has been reported as a cause for bronchiolitis in microwave popcorn manufacturing workers. ${ }^{34,35}$ Despite the knowledge of this association between diacetyl and lung injury, a recent study identified its presence in 110 out of 159 tested "sweet" e- liquids. ${ }^{36,37}$

The e-cigarette metal coils have been reported to contain variable amounts of chromium, manganese, nickel, lead, copper, silver, zinc, and tin and the wicks can be made of silica. ${ }^{38,39}$ There are concerns that the higher heat settings that some devices offer can lead to the evolution of nanoparticles of these various metals $^{38}$ (see article abstract below) and acrolein ${ }^{15}$ (a known carcinogen) from the various additives in the e-liquids. There also have been several reports of these devices exploding and causing major injuries. ${ }^{40}$

Hence, while e-cigarettes may indeed help some individuals to transition from the use of tobacco smoking to nicotine vaping, there is growing evidence that the ingredients, including nicotine itself, may have significant adverse health consequences. Yet there is very little information about the consequences of long-term use of these devices and they remain largely unregulated in terms of content, labeling and access.

The first paper reviewed in this Journal Club is from the European Respiratory Society (ERS) Tobacco Control Committee Statement regarding e-cigarettes. The Taskforce cautions that tobacco harm reduction strategies that endorse the use of alternative nicotine delivery products for smoking cessation have not been proven to be as or more effective than traditional smoking cessation strategies and have been based upon incorrect assumptions and unfounded or undocumented claims. ${ }^{13}$ The ERS Statement emphasizes that there is lack of proof supporting claims that nicotine delivery devices such as e-cigarettes and "heated not burned" tobacco products (which eliminates combustible particulates and other components that evolve from the high heat of the actual burning of tobacco cigarettes) are efficacious in helping people to quit smoking permanently and in fact there is growing evidence that they are actually harmful to health. The Taskforce urges policymakers and public health bodies to reevaluate smoking cessation programs that involve 
the use of e-cigarettes and heated tobacco products and states that the Taskforce cannot recommend this approach as an effective population-based strategy.

At the most recent American Thoracic Society International Conference in Dallas, Texas in May of this year, there were numerous posters and lectures on e-cigarettes and a large scientific symposium entitled, "Addicting a New Generation: JUULing, Vaping, Heat Not Burn, Flavorings and the Evidence for Why We Should Be Very Concerned." Many of the topics outlined above were covered and the organizers emphasized the critical need for further research in several different areas.

In this Journal Club we review the ERS task force report and some of the most recent studies looking at e-cigarettes as a method of harm reduction in smoking cessation, the potential that these new devices may actually have significant health effects, the likelihood of e-cigarettes leading to never tobacco smokers becoming tobacco smokers and concerns regarding the urgent need for research studies that can lead to informed and appropriate subsequent policy and regulation of these products.

Note: Abstracts are presented in their original, published format and have not been edited to match JCOPDF style.

\section{Abstract 1 \\ Electronic Cigarettes: A Task Force Report from the European Respiratory Society}

\author{
Bals R, Boyd J, Esposito S, et al. Eur Respir J. \\ 2019;53(2):1801151. \\ doi: https://doi.org/10.1183/13993003.01151-2018
}

There is a marked increase in the development and use of electronic nicotine delivery systems or electronic cigarettes (ECIGs). This statement covers electronic cigarettes (ECIGs), defined as "electrical devices that generate an aerosol from a liquid" and thus excludes devices that contain tobacco. Database searches identified published articles that were used to summarize the current knowledge on the epidemiology of ECIG use; their ingredients and accompanied health effects; second-hand exposure; use of ECIGs for smoking cessation; behavioral aspects of ECIGs and social impact; in vitro and animal studies; and user perspectives. ECIG aerosol contains potentially toxic chemicals. As compared to conventional cigarettes, these are fewer and generally in lower concentrations. Second-hand exposures to ECIG chemicals may represent a potential risk, especially to vulnerable populations. There is not enough scientific evidence to support ECIGs as an aid to smoking cessation due to a lack of controlled trials, including those that compare ECIGs with licenced stop-smoking treatments. So far, there are conflicting data that use of ECIGs results in a renormalisation of smoking behaviour or for the gateway hypothesis. Experiments in cell cultures and animal studies show that ECIGs can have multiple negative effects. The long-term effects of ECIG use are unknown, and there is therefore no evidence that ECIGs are safer than tobacco in the long term. Based on current knowledge, negative health effects cannot be ruled out.

\section{Comments}

The ERS Task Force report published in January of 2019 is an excellent distillation of the best and most relevant studies published up to 2016. Members of the Task Force were from Europe and the United States and they reviewed 2271 papers. There are over 290 references in the publication. While it is not a systematic literature review per se, the information is organized into several themes including epidemiology, design and operation of the devices, animal and human studies on health effects and toxicity of the various and variable constituents of e-cigarettes as well as the potential concerns about secondhand exposure. It also reviews the literature regarding e-cigarettes as a tool for smoking cessation and the behavioral and social impacts of e-cigarettes such as serving as a gateway for people to become tobacco smokers and the renormalization of smoking in public. It is an excellent introduction to the field with excellent critique of the quality of studies and the key research questions that need to be addressed for each area. It provides an excellent framework of how to evaluate e-cigarettes going forward and most importantly presents a cogent argument for the need for further research to inform the proper utilization and regulation of e-cigarettes. 


\section{Abstract 2 \\ A Randomized Trial of E-Cigarettes Versus Nicotine-Replacement Therapy}

\author{
Hajek P, Phillips-Waller A, Przulj D, et al. $N$ Engl $J$ \\ Med. 2019;380(7):629-637. \\ doi: https://doi.org/10.1056/NEJMoa 1808779
}

\section{BACKGROUND:}

E-cigarettes are commonly used in attempts to stop smoking, but evidence is limited regarding their effectiveness as compared with that of nicotine products approved as smoking-cessation treatments.

\section{METHODS:}

We randomly assigned adults attending U.K. National Health Service stop-smoking services to either nicotine-replacement products of their choice, including product combinations, provided for up to 3 months, or an e-cigarette starter pack (a secondgeneration refillable e-cigarette with one bottle of nicotine e-liquid [18 mg per milliliter]), with a recommendation to purchase further e-liquids of the flavor and strength of their choice. Treatment included weekly behavioral support for at least 4 weeks. The primary outcome was sustained abstinence for 1 year, which was validated biochemically at the final visit. Participants who were lost to follow-up or did not provide biochemical validation were considered to not be abstinent. Secondary outcomes included participant-reported treatment usage and respiratory symptoms.

\section{RESULTS:}

A total of 886 participants underwent randomization. The 1-year abstinence rate was $18.0 \%$ in the e-cigarette group, as compared with $9.9 \%$ in the nicotine-replacement group (relative risk, 1.83; 95\% confidence interval [CI], 1.30 to 2.58; $\mathrm{P}<0.001$ ). Among participants with 1-year abstinence, those in the e-cigarette group were more likely than those in the nicotine-replacement group to use their assigned product at 52 weeks (80\% [63 of 79 participants] vs. $9 \%$ [ 4 of 44 participants]). Overall, throat or mouth irritation was reported more frequently in the e-cigarette group (65.3\%, vs. $51.2 \%$ in the nicotine- replacement group) and nausea more frequently in the nicotine-replacement group (37.9\%, vs. $31.3 \%$ in the e-cigarette group). The e-cigarette group reported greater declines in the incidence of cough and phlegm production from baseline to 52 weeks than did the nicotine-replacement group (relative risk for cough, 0.8 ; $95 \%$ CI, 0.6 to 0.9 ; relative risk for phlegm, 0.7; 95\% CI, 0.6 to 0.9). There were no significant between-group differences in the incidence of wheezing or shortness of breath.

\section{CONCLUSIONS:}

E-cigarettes were more effective for smoking cessation than nicotine-replacement therapy, when both products were accompanied by behavioral support. (Funded by the National Institute for Health Research and Cancer Research UK; Current Controlled Trials number, ISRCTN60477608

\section{Comments}

This is one of the most rigorous studies to demonstrate the potential benefit of e-cigarettes as a smoking cessation tool. Overall the abstinence results are fairly low for both the e-cigarette and nicotine replacement therapy groups, but it is almost twice as high for the e-cigarette group. It is worth noting that participants were not blinded in the study, but they had to claim no preference of one type of treatment over the other in order to enter the trial. Nonetheless, up to $75 \%$ of the participants had previously tried and failed to quit using nicotine replacement therapy in the past. This may select a group that is less responsive or has greater side effects and lower expectations from standard smoking cessation strategies and hence may not try to stay in trial. The nicotine replacement group did not have the option to use bupropion which has been shown to be more effective than nicotine replacement therapy alone especially for those with a history of depression. ${ }^{41-43}$ There is a study planned that will be a double-blind double dummy trial comparing e-cigarettes versus varenicline; the protocol has been recently published. ${ }^{44}$ It is also worth noting that most of the e-cigarette group participants were responsible for buying further refills after the initial starter pack. They could pick different devices and different flavors and different strengths of e-liquids after they used up the starter kit. The provided devices were also only second 
generation and lacked the variable controls of some 3rd and 4th generation devices. The newer devices are able to produce higher exposures to nicotine and other components and consequently potential side effects, (see Lechasseur et al abstract below). ${ }^{38}$ Interestingly, more of the e-cigarette group reported serious respiratory adverse events but the e-cigarette group did have a lower respiratory infection rate. As the authors point out, while this study has relevance for the group of current nicotine dependent individuals who may be looking at using e-cigarettes to quit smoking, it is not likely generalizable to the population of smokers who are less dependent or those who try e-cigarettes for reasons besides smoking cessation. Further, it remains to be seen how generalizable the findings are considering that it was conducted within the United Kingdom National Health Stop Smoking Service setting and variable in terms of participants likely to utilize the service and the overall nature of the levels of support in the program.

\section{Abstract 3 \\ Nicotine Matters in Predicting Subsequent Smoking After E-Cigarette Experimentation: A Longitudinal Study Among Finnish Adolescents}

Kinnunen JM, Ollila H, Minkkinen J, Lindfors PL, Timberlake DS, Rimpelä AH. Drug Alcohol Depend. 2019;201:182-187.

doi: https://doi.org/10.1016/j.drugalcdep.2019.04.019

\section{BACKGROUND:}

Several studies indicate an association between e-cigarette use and subsequent smoking among youth. However, most previous studies lack measures of the nicotine content of e-liquid and have not usually measured regular smoking.

\section{METHODS:}

We tested the association between e-cigarette use, with and without nicotine, and subsequent daily use of conventional cigarettes and nicotine e-cigarettes among study population of 3474 students. A surveywas conducted in lower secondary schools of the Helsinki metropolitan area, Finland, with 15 - 16-year-olds in 2014 (baseline) and in upper secondary schools in
2016 when the cohort was 17 - 18-year-olds (followup). Firth logistic regression and generalized linear mixed models (GLMM) were used.

\section{RESULTS:}

Of students, $25 \%$ had experimented with nicotine e-cigarettes at baseline and $40 \%$ at follow-up. Among baseline never-smokers, experimentation with or use of nicotine e-cigarettes predicted the uptake of daily smoking at follow-up (AOR 2.92; 95\% CI 1.09-7.85), but baseline experimentation with nonnicotine e-cigarettes did not when compared with the non-e-cigarette experimenters. Nicotine e-cigarette experimentation at baseline predicted daily nicotine e-cigarette use at follow-up (AOR 2.96; 95\% CI 1.227.22). Non-nicotine e-cigarette experimentation at baseline did not predict statistically significantly daily nicotine e-cigarette use at follow-up (AOR 3.13; 95\% CI 0.98-10.02). The small number of cases may have diminished the statistical significance.

\section{CONCLUSIONS:}

The findings suggest that experimentation with nicotine e-cigarettes serves as a gateway to subsequent use of conventional cigarettes as well as nicotine e-cigarettes. Our results support the actions to limit youths' access to e-cigarettes in order to prevent nicotine addiction.

\section{Comments}

This is one of the largest cohorts that have been followed longitudinally and addresses a very important question. The Hajek et al study discussed above demonstrates that even if we accept the possibility that use of e-cigarettes for smoking cessation may have some slight advantages over conventional smoking cessation interventions, there is a much bigger concern about promoting the use of these devices long term as they may become a gateway for nicotine naïve adolescents to become long-term users of these devices or move on to smoke conventional cigarettes and beyond. 


\section{Abstract 4 \\ E-Cigarette Use Causes a Unique Innate Immune Response in the Lung, Involving Increased Neutrophilic Activation and Altered Mucin Secretion}

Reidel B, Radicioni G, Clapp PW, et al. Am J Respir Crit Care Med. 2018;197(4):492-501.

doi: https://doi.org/10.1164/rccm.201708-15900C

\section{RATIONALE:}

E-cigarettes have become increasingly popular and little is known about their potential adverse health effects.

\section{OBJECTIVES:}

To determine the effects of e-cigarette use on the airways.

\section{METHODS:}

Induced sputum samples from cigarette smokers, e-cigarette users, and nonsmokers were analyzed by quantitative proteomics, and the total and individual concentrations of mucins MUC5AC and MUC5B were determined by light scattering/refractometry and labeled mass spectrometry, respectively. Neutrophil extracellular trap (NET) formation rates were also determined for the same groups.

\section{MEASUREMENTS AND MAIN RESULTS:}

E-cigarette users exhibited significant increases in aldehyde-detoxification and oxidative stressrelated proteins associated with cigarette smoke compared with nonsmokers. The levels of innate defense proteins associated with chronic obstructive pulmonary disease, such as elastase and matrix metalloproteinase-9, were significantly elevated in e-cigarette users as well. E-cigarette users' sputum also uniquely exhibited significant increases in neutrophil granulocyte-related and NET-related proteins, such as myeloperoxidase, azurocidin, and protein-arginine deiminase 4, despite no significant elevation in neutrophil cell counts. Peripheral neutrophils from e-cigarette users showed increased susceptibility to phorbol 12-myristate 13-acetate-inducosis. Finally, a compositional change in the gel-forming building blocks of airway mucus (i.e., an elevated concentration of mucin MUC5AC) was observed in both cigarette smokers and e-cigarette users.

\section{CONCLUSIONS:}

Together, our results indicate that e-cigarette use alters the profile of innate defense proteins in airway secretions, inducing similar and unique changes relative to cigarette smoking. These data challenge the concept that e-cigarettes are a healthier alternative to cigarettes.

\section{KEYWORDS:}

NET; e-cigarette; lung; mucin; neutrophil

\section{Comments}

The findings from this study and others have raised serious concerns about the possible long-term health effects of e-cigarette use as a substitute for cigarette smoking rather than as a short- term alternative/ bridge for smoking cessation. This is particularly important when considering already compromised individuals such as COPD patients and COPD/asthma overlap patients. ${ }^{45}$ It reinforces the need for further longitudinal studies and the prudence of regulating these devices and e-liquids.

\section{Abstract 5 \\ Variations in Coil Temperature/ Power and E-Liquid Constituents Change Size and Lung Deposition of Particles Emitted by an Electronic Cigarette}

Lechasseur A, Altmejd S, Turgeon N, et al. Physiol Rep. 2019;7(10):e14093.

doi: https://doi.org/10.14814/phy2.14093

Electronic cigarette uses propylene glycol and glycerol to deliver nicotine and flavors to the lungs. Given the hundreds of different brands, the thousands of flavors available and the variations in nicotine concentrations, it is likely that electronic cigarette settings and e-liquid composition affect the size distribution of particles emitted and ultimately pulmonary deposition. We used the inExpose e-cigarette extension to study two separate modes 
of operation of electronic cigarettes, namely powercontrolled and the temperature-controlled. We also assessed several e-liquids based on propylene glycol and glycerol concentrations, nicotine content, and selected monomolecular flavoring agents (menthol, vanillin, and maltol). Particle size distribution was measured using a Condensation Particle Counter and a Scanning Mobility Particle Sizer spectrometer. Lung deposition was predicted using the International Commission on Radiological Protection model. For all resistance coils, increase in power delivery generated larger particles while maintaining a higher coil temperature generated smaller particles. Increase in glycerol concentration led to the generation of larger particles. With regard to flavors, we showed that despite minor effect of menthol and maltol, vanillin dramatically increased particle size. Presence of nicotine also increased particle size. Finally, particles emitted by the electronic cigarette were predicted to mainly deposit in the alveoli and conditions generating larger particle sizes led to a reduction in predicted lung deposition. This study shows that coil temperature, propylene glycol and glycerol concentrations, presence of nicotine, and flavors affect the size of particles emitted by an electronic cigarette, directly affecting predicted lung deposition of these particles.

○2019 The Authors. Physiological Reports published by Wiley Periodicals, Inc. on behalf of The Physiological Society and the American Physiological Society.

\section{KEYWORDS:}

Electronic cigarette; e-liquid; lung distribution; nicotine; particle size; vaping

\section{Comments}

As discussed in the review of the Hajek paper, the newer generation devices that afford greater ability for the user to modulate the aerosol generated can result in highly variable content and nature of the aerosol that is generated leading to differences in the deposition and possibly the health consequences.

\section{Abstract 6 The Rise of E-Cigarettes, Pod Mod Devices, and JUUL Among Youth: Factors Influencing Use, Health Implications, and Downstream Effects}

Fadus MC, Smith TT Squeglia LM. Drug Alcohol Depend. 2019;201:85-93.

doi: https://doi.org/10.1016/j.drugalcdep.2019.04.011

\section{BACKGROUND:}

Electronic cigarettes (e-cigarettes) were first introduced in the U.S. market in 2006, with the more recent evolution of "pod-mod" e-cigarettes such as JUUL introduced in 2015. Although marketed as a smoking cessation tool, e-cigarettes are rarely used for this purpose in youth. This review aims to synthesize the literature regarding e-cigarette use among youth, and provides a resource for clinicians, educators, and families that helps answer commonly asked questions about e-cigarettes.

\section{METHODS:}

PubMed, Scopus, and PsycINFO search was performed using search terms "Electronic Nicotine Delivery Systems," "e cigarettes," "e-cigarettes," "electronic cigarettes," "vaping," “JUUL," "e-cigs," and "vape pens." Search results were filtered to only include those related to adolescents and young adults.

\section{RESULTS:}

E-cigarette use among youth is common, with rates of use increasing from $1.5 \%$ in 2011 to $20.8 \%$ in 2018. Pod mod devices such as JUUL have gained favor among youth for their sleek design, userfriendly function, desirable flavors, and ability to be used discreetly in places where smoking is forbidden. Adolescents are often uninformed about the constituents of e-cigarettes, and little is known about the long-term effects of e-cigarettes. Studies have suggested a "gateway" effect for combustible cigarettes and cannabis use.

\section{CONCLUSIONS:}

E-cigarette use is becoming increasingly common among youth, leading to a myriad of questions and concerns from providers, educators, and family 
members. More research is needed to determine the ultimate public health impact of e-cigarette use. The authors provide a summary table of frequently asked questions in order to help clarify these common concerns.

\section{Comments}

JUUL has been the leading retailer of e-cigarette products in the United States and has developed the POD system of sleekly designed e-cigarettes that look like USB devices. In early July of this year, San Francisco, home of JUUL's headquarters, imposed a city-wide ban on the sale of all vaping/ecigarette products until they undergo Food and Drug Administration (FDA) reviews. Unfortunately, at the current time, the FDA has stated that e-cigarette companies have until 2022 before they will have to apply for regulatory approval. This currently is being challenged in court. It is worth noting that the JUUL website now restricts sales to those 21 years and above and potential buyers must agree to an age verification step (photo ID), and upload a photo that is analyzed using face recognition ID. They also state they have a "youth prevention effort" in place. In their statement they claim that they don't want anyone who doesn't smoke or already use nicotine to use JUUL products. The sentence, "we certainly don't want youth using the product" is in this statement. They have also removed certain fruit flavors from retail distribution. This plan was initiated in November 2018.

\section{Abstract 7 \\ High-Nicotine Electronic Cigarette Products: Toxicity of JUUL Fluids and Aerosols Correlates Strongly with Nicotine and Some Flavor Chemical Concentrations}

Omaiye EE, McWhirter KJ, Luo W, Pankow JF, Talbot P. Chem Res Toxicol. 2019;32(6):1058-1069.

doi: https://doi.org/10.1021/acs.chemrestox.8b00381

Whereas JUUL electronic cigarettes (ECs) have captured the majority of the EC market, with a large fraction of their sales going to adolescents, little is known about their cytotoxicity and potential effects on health. The purpose of this study was to determine flavor chemical and nicotine concentrations in the eight currently marketed prefilled JUUL EC cartridges ("pods") and to evaluate the cytotoxicity of the different variants (e.g., "Cool Mint" and "Crème Brulee") using in vitro assays. Nicotine and flavor chemicals were analyzed using gas chromatographymass spectrometry in pod fluid before and after vaping and in the corresponding aerosols. 59 flavor chemicals were identified in JUUL pod fluids, and 3 were $>1 \mathrm{mg} / \mathrm{mL}$. Duplicate pods were similar in flavor chemical composition and concentration. Nicotine concentrations (average $60.9 \mathrm{mg} / \mathrm{mL}$ ) were significantly higher than those of any EC products we have previously analyzed. The transfer efficiency of individual flavor chemicals that were $>1 \mathrm{mg} / \mathrm{mL}$ and nicotine from the pod fluid into aerosols was generally $35-80 \%$. All pod fluids were cytotoxic at a 1:10 dilution (10\%) in the MTT and neutral red uptake assays when tested with BEAS-2B lung epithelial cells. Most aerosols were cytotoxic in these assays at concentrations between 0.2 and $1.8 \%$. The cytotoxicity of collected aerosol materials was highly correlated with nicotine and ethyl maltol concentrations and moderately to weakly correlated with total flavor chemical concentration and menthol concentration. Our study demonstrates that (1) some JUUL flavor pods have sufficiently high concentrations of flavor chemicals that may make them attractive to youth and (2) the concentrations of nicotine and some flavor chemicals (e.g., ethyl maltol) are high enough to be cytotoxic in acute in vitro assays, emphasizing the need to determine if JUUL products will lead to adverse health effects with chronic use

\section{Comments}

This study highlights that the high levels of nicotine and flavoring agents that are being used in these devices may attract primarily young users and be highly addicting. They now sell a POD/cartridge that has a $59 \mathrm{mg} / \mathrm{ml}$ concentration that is purported to have the nicotine equivalent of 1 pack of cigarettes. ${ }^{46}$ Each JUUL pod or cartridge contains $0.7 \mathrm{ml}$ of liquid with about $45 \mathrm{mg}$ of nicotine (or $50 \mathrm{mg}$ nicotine salt) for a concentration of $59 \mathrm{mg} / \mathrm{ml}$ of liquid. This is supposed to provide up to the equivalent of 200 puffs. JUUL was the first producer to incorporate the use of nicotine salts and benzoic acid which increases 
the bioavailability of nicotine without causing the irritation to the back of the throat that a liquid form of nicotine would cause. It is important to note that even though each POD contains the equivalent of 1 pack of cigarettes the user is not obliged to use the contents of a POD all at one time. The user can stretch the use of a single POD out over time if so desired.

\section{Bottom Line}

While it is true that, relatively speaking, e-cigarettes have fewer potential toxic compounds than regular tobacco cigarettes, there is mounting evidence that they are not benign and may indeed have health effects that are not inconsequential. In fact, some individuals with the greatest incentives to stop smoking, COPD and/or asthma patients, may be more susceptible to the potential harmful effects of e-cigarettes. Furthermore, e-cigarettes are no longer being marketed solely as part of a temporary smoking cessation strategy but rather as an alternative nicotine delivery device that may be intended for long-term consumption.
Particularly alarming is the evidence that substantial numbers of young people are trying e-cigarettes, and some are moving on to smoking tobacco cigarettes and other agents. Recently, various states and cities have already banned sales and imposed age restrictions, licensing requirements, taxation of e-cigarettes, indoor vaping bans, and banning of certain flavorings that may target youth until further research provides answers that will allow an informed and rational policy formulation and appropriate regulation. The ERS and the Forum of International Respiratory Societies have issued position statements on e- cigarettes stating that they cannot endorse these products as part of a public health "harm reduction" policy based on the evidence available at current time. They emphasize the critical need for more independent studies regarding the benefits and potential harms of e-cigarette use and a prudent restriction of the usage of these products at least until their safety and efficacy can be established. Hopefully other prominent respiratory societies globally will follow suit and render similar position statements. 


\section{References}

1. Chun LF, Moazed F, Caffee CS, Matthay MA, Gotts JF. Pulmonary toxicity of e-cigarettes. Am J Physiol Lung Cell Mol Physiol. 2017;313(2):193-206.

doi: https://doi.org/10.1152/ajplung.00071.2017

2. Soneji SS, Knutzen KE, Villanti AC. Use of flavored e-cigarettes among adolescents, young adults, and older adults: findings from the population assessment for tobacco and health study. Public Health Rep. 2019;134(3):282-292. doi: https://doi.org/10.1177/0033354919830967

3. Fadus MC, Smith TT, Squeglia LM. The rise of e-cigarettes, pod mod devices, and JUUL among youth: factors influencing use, health implications, and downstream effects. Drug Alcohol Depend. 2019;201:85-93.

doi: https://doi.org/10.1016/j.drugalcdep.2019.04.011

4. Gottlieb MA. Regulation of e-cigarettes in the United States and its role in a youth epidemic. Children (Basel). 2019;6(3):40. doi: https://doi.org/10.3390/children6030040

5. Hallingberg B, Maynard OE, Bauld L, et al. Have e-cigarettes renormalised or displacedyouth smoking? Results of a segmented regression analysis of repeated cross-sectional survey data in England, Scotland and Wales. Tob Control. 2019; April 1 online first. doi: https://doi.org/10.1136/tobaccocontrol-2018-054584

6. Jenssen BP, Boykan R. Electronic cigarettes and youth in the United States: a call to action (at the local, national and logbal levels). Children (Basel). 2019;6(2):30.

doi: https://doi.org/10.3390/children6020030

7. Klein JD. E-cigarettes: a 1-way street to traditional smoking and nicotine addiction for youth. Pediatrics. 2018;141(1).

doi: https://doi.org/10.1542/peds.2017-2850

8. Krishnan-Sarin S, Green BG, Kong G, et al. Studying the interactive effects of menthol and nicotine among youth: an examination using e-cigarettes. Drug Alcohol Depend. 2017;180:193-199.

doi: https://doi.org/10.1016/j.drugalcdep.2017.07.044

9. Darville A, Hahn EJ. E-cigarettes and atherosclerotic cardiovascular disease: what clinicians and researchers need to know. Curr Atheroscler Rep. 2019;21(5):15. doi: https://doi.org/10.1007/s11883-019-0777-7

10. Skotsimara G, Antonopoulos AS, Oikonomou E, et al. Cardiovascular effects of electronic cigarettes: a systematic review and meta-analysis. Eur J Prev Cardiol. 2019; 26(11): 12191228. doi: https://doi.org/10.1177/2047487319832975

11. Wang JB, Olgin JE, Nah G, et al Cigarette and e-cigarette dual use and risk of cardiopulmonary symptoms in the Health eHeart Study. PLoS One. 2018;13(7): e0198681. doi: https://doi.org/10.1371/journal.pone.0198681
12. Kesimer M. Another warning sign: high nicotine content in e-cigarettes disrupts mucociliary clearance, the essential defense mechanism of the lung. Am J Respir Crit Care Med. 2019; June 14 online first.

doi: https://doi.org/10.1164/rccm.201905-1080ED

13. Bals R, Boyd J, Esposito S, et al. Electronic cigarettes: a task force report from the European Respiratory Society. Eur Respir J. 2019; 53(2): 1801151.

doi: https://doi.org/10.1183/13993003.01151-2018

14. Thirion-Romero I, Perez-Padilla R, Zabert G, BarrientosGutierrez. Respiratory impact of electronic cigarettes and "lowrisk" tobacco. Rev Invest Clin. 2019;71(1):17-27.

doi: https://doi.org/10.24875/RIC.18002616

15. Stephens WE. Comparing the cancer potencies of emissions from vapourised nicotine products including e-cigarettes with those of tobacco smoke. Tob Control. 2017; 27:10-17. doi: https://doi.org/10.1136/tobaccocontrol-2017-053808

16. Haussmann HJ, Fariss MW. Comprehensive review of epidemiological and animal studies on the potential carcinogenic effects of nicotine per se. Crit Rev Toxicol. 2016; 46(8):701-734.

doi: https://doi.org/10.1080/10408444.2016.1182116

17. Bozier J, Rutting S, Xenaki P, Peters M, Adcock I, Oliver BG. Heightened response to e-cigarettes in COPD. ERJ Open Res. 2019;5(1):00192-2018.

doi: https://doi.org/10.1183/23120541.00192-2018

18. Higham A, Bostock D, Booth G, Dungra JV, Singh D. The effect of electronic cigarette and tobacco smoke exposure on COPD bronchial epithelial cell inflammatory responses. Int J Chron Obstruct Pulmon Dis. 2018;13: 989-1000.

doi: https://doi.org/10.2147/COPD.S157728

19. Ahmad S, Zafar I, Mariappan N, et al. Acute pulmonary effects of aerosolized nicotine. Am J Physiol Lung Cell Mol Physiol. 2019; 316(1):L94-L104.

doi: https://doi.org/10.1152/ajplung.00564.2017

20. Omaiye EE, McWhirter KJ, Luo W, Pankow JF, Talbot P. Highnicotine electronic cigarette products: toxicity of JUUL fluids and aerosols correlates strongly with nicotine and some flavor chemical concentrations. Chem Res Toxicol. 2019;32(6):10581069. doi: https://doi.org/10.1021/acs.chemrestox.8b00381

21. Chapman S, Bareham D, Maziak W. The gateway effect of e-cigarettes: reflections on main criticisms. Nicotine Tob Res. 2019;21(5):695-698. doi: https://doi.org/10.1093/ntr/nty067

22. Bowler RP, Hansel NN, Jacobson S, et al. Electronic cigarette use in U.S. adults at risk for or with COPD: analysis from two observational cohorts. J Gen Intern Med. 2017;32(12):1315-1322. doi: https://doi.org/10.1007/s11606-017-4150-7

23. Pourchez J, Forest V. E-cigarettes: from nicotine to cannabinoids, the French situation. Lancet Respir Med. 2018;6(5): e16. doi: https://doi.org/10.1016/S2213-2600(18)30069-9 
24. Farsalinos K, Poulas K, Voudris V. Changes in puffing topography and nicotine consumption depending on the power setting of electronic cigarettes. Nicotine Tob Res. 2018;20(8):993-997. doi: https://doi.org/10.1093/ntr/ntx219

25. Fearon IM, Eldridge AC, Gale N, McEwan M, Stiles MF, Round EK. Nicotine pharmacokinetics of electronic cigarettes: A review of the literature. Regul Toxicol Pharmacol. 2018;100:25-34. doi: https://doi.org/10.1016/j.yrtph.2018.09.004

26. Kosmider L, Spindle TR, Gawron M, Sobczak A, Geniewicz ML. Nicotine emissions from electronic cigarettes: Individual and interactive effects of propylene glycol to vegetable glycerin composition and device power output. Food Chem Toxicol. 2018;115:302-305. doi: https://doi.org/10.1016/j.fct.2018.03.025

27. Perkins KA. FDA policy on setting maximum nicotine content in cigarettes. Nicotine Tob Res. 2019;21(7):996-997. doi: https://doi.org/10.1093/ntr/nty068

28. St Helen G, Dempsey DA, Havel CM, Jacob P III. Impact of e-liquid flavors on nicotine intake and pharmacology of e-cigarettes. Drug Alcohol Depend. 2017;178:391-398. doi: https://doi.org/10.1016/j.drugalcdep.2017.05.042

29. Bono RS, Barnes AJ, Lester RC, Cobb CO. Effects of electronic cigarette liquid flavors and modified risk messages on perceptions and subjective effects of e-cigarettes. Health Educ Behav. 2019;46(2): 197-203.

doi: https://doi.org/10.1177/1090198118806965

30. Lee MS, LeBouf RK, San Y-S, Koutrakis P, Christiani DC. Nicotine, aerosol particles, carbonyls and volatile organic compounds in tobacco- and menthol-flavored e-cigarettes. Environ Health. 2017;16(1):42. doi: https://doi.org/10.1186/s 12940-017-0249-x

31. Aokage T, Tsukahara K, Fukuda Y, et al. Heat-not-burn cigarettes induce fulminant acute eosinophilic pneumonia requiring extracorporeal membrane oxygenation. Respir Med Case Rep. 2019;26:87-90. doi: https://doi.org/10.1016/j.rmcr.2018.12.002

32. Arter ZL, Wiggins A, Hudspath C, Kisling A, Hostler DC, Hostler JM. Acute eosinophilic pneumonia following electronic cigarette use. Respir Med Case Rep. 29;27:100825.

doi: https://doi.org/10.1016/j.rmcr.2019.100825

33. Itoh M, Aoshiba K, Herai Y, Nakamura H, Takemura T. Lung injury associated with electronic cigarettes inhalation diagnosed by transbronchial lung biopsy. Respirol Case Rep. 2018;6(1):e00282. doi: https://doi.org/10.1002/rcr2.282

34. Lockey JE, Hilbert TJ, Levin PH, et al. Airway obstruction related to diacetyl exposure at microwave popcorn production facilities. Eur Respir J. 2009;34(1):63-71. doi: https://doi.org/10.1183/09031936.00050808

35. Park RM, Gilbert SJ. Pulmonary impairment and risk assessment in a diacetyl-exposed population: microwave popcorn workers. $J$ Occup Environ Med. 2018;60(6): 496-506. doi: https://doi.org/10.1097/JOM.0000000000001303
36. Farsalinos KE, Gilman IG, Melvin MS, et al. Nicotine levels and presence of selected tobacco-derived toxins in tobacco flavoured electronic cigarette refill liquids. Int J Environ Res Public Health. 2015;12(4):3439-3452.

doi: https://doi.org/10.3390/ijerph120403439

37. Farsalinos KE, Kistler KA, Gillman G, Voudris V. Evaluation of electronic cigarette liquids and aerosol for the presence of selected inhalation toxins. Nicotine Tob Res. 2015;17(2):168-174. doi: https://doi.org/10.1093/ntr/ntu176

38. Lechasseur A, Altmejd S, Turgeon N, et al. Variations in coil temperature/power and e-liquid constituents change size and lung deposition of particles emitted by an electronic cigarette. Physiol Rep. 2019;7(10):e14093. doi: https://doi.org/10.14814/phy2.14093

39. Williams M, To A, Bozhilov K, Talbot P. Strategies to reduce tin and other metals in electronic cigarette aerosol. PLoS One. 2015;10(9):e0138933.

doi: https://doi.org/10.1371/journal.pone.0138933

40. Brownson EG, Thompson CM, Goldsberry S, et al. Explosion injuries from e-cigarettes. N Engl J Med. 2016;375(14):14001402. doi: https://doi.org/10.1056/NEJMc1608478

41. Kotz D, Simpson C, Viechtbauer W, van Schayck OCP, West $\mathrm{R}$, Sheikh A. Cardiovascular and neuropsychiatric safety of varenicline and bupropion compared with nicotine replacement therapy for smoking cessation: study protocol of a retrospective cohort study using the QResearch general practice database. BMJ Open. 2014;4(8):e005281.

doi: https://doi.org/10.1136/bmjopen-2014-005281

42. Lamarche K. Review: varenicline, bupropion, and nicotine replacement therapies are effective for smoking cessation at 6 or 12 months. Evid Based Nurs. 2009;12(1):10.

doi: https://doi.org/10.1136/ebn.12.1.10

43. Stapleton J, West R, Hajek P, et al. Randomized trial of nicotine replacement therapy (NRT), bupropion and NRT plus bupropion for smoking cessation: effectiveness in clinical practice. Addiction. 2013;108(12): 2193-2201.

doi: https://doi.org/10.1111/add.12304

44. Berlin I, Dautzenberg B, Lehman B, et al. Randomised, placebocontrolled, double-blind, double-dummy, multicentre trial comparing electronic cigarettes with nicotine to varenicline and to electronic cigarettes without nicotine: the ECSMOKE trial protocol. BMJ Open. 2019; 9(5): e028832. doi: https://doi. org/10.1136/bmjopen-2018-028832

45. Clapp PW, Jaspers I. Electronic cigarettes: their constituents and potential links to asthma. Curr Allergy Asthma Rep. 2017;17(11):79. doi: https://doi.org/10.1007/s11882-017-0747-5

46. Jackler RK, Ramamurthi D. Nicotine arms race: JUUL and the high-nicotine product market. Tob Control. 2019; online first: 06 Feb 2019.

doi: https://doi.org/10.1136/tobaccocontrol-2018-054796 